\title{
Barriers to carriers: faults and recombination in non- stoichiometric perovskite scintillators
}

\author{
Anjana Talapatra ${ }^{1, \star}$, Dibyajyoti Ghosh ${ }^{2,3}$, Blas P. Uberuaga ${ }^{1}$ (D), and Ghanshyam Pilania ${ }^{1}$ \\ ${ }^{7}$ Materials Science and Technology Division, Los Alamos National Laboratory, Los Alamos, NM 87545, USA \\ ${ }^{2}$ Theoretical Division, Los Alamos National Laboratory, Los Alamos, NM 87545, USA \\ ${ }^{3}$ Center for Nonlinear Studies, Los Alamos National Laboratory, Los Alamos, NM 87545, USA
}

Received: 12 April 2021

Accepted: 22 June 2021

Published online:

16 July 2021

(C) The Author(s) 2021

\begin{abstract}
Tuning the efficiency and speed of charge carrier recombination in inorganic scintillators can potentially improve their performance in diverse applications. Recent work suggests that this maybe be achieved via a two-phase scintillator $A B$ that naturally phase separates into $A$-rich and $B$-rich domains. In addition, a favorable electronic structure and band-edge alignment such that the charge carriers are confined or are thermodynamically driven to preferentially accumulate in one of the two domains, might lead to an improved radiative recombination rate. Here, we use density functional theory computations and ab initio molecular dynamics (AIMD), including non-adiabatic molecular dynamics (NAMD) simulations, to examine an alternative phase structure and its potential impact on recombination. Using a model perovskite $\mathrm{SrTiO}_{3}$ system with one-, two- and three-dimensional Ruddlesden-Popper (RP) phases, we demonstrate that RP faults induce band structure changes in the material that can act as barriers to carrier transport. Our AIMD/NAMD simulations indicate competing effects of a lower mean free path (potentially enhancing the desired radiative recombination and overall scintillating efficiency) and faster non-radiative recombination (undesired) due to enhanced electron-phonon coupling in the faulted system. Full exploitation of such a rational design approach would require tuning of the effective scintillation efficiency by varying the perovskite chemistry using appropriate arrangements of RP faults in the bulk material. Finally, other effects, such as the tendency of point defects to segregate at the interface, that might affect the overall performance, are briefly discussed. We expect the basic results found here to apply to other nanostructured scintillators.
\end{abstract}

Handling Editor: Pedro Camargo.

Address correspondence to E-mail: atalapatra@lanl.gov 


\section{GRAPHICAL ABSTRACT}

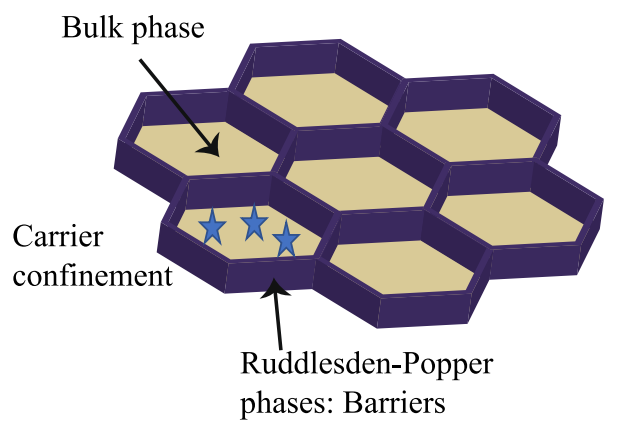

\section{Introduction}

Scintillators, materials that convert the kinetic energy of high energy particles into low energy photons that are more readily detected, are central to a number of applications where imaging is important. By converting, for example, high-energy $\mathrm{X}$-rays to visible light, these materials are integral components in X-ray diagnostic machines that can visualize the internals of a range of objects, from the human body to materials of interest in high energy physics experiments. Thus, improvements in scintillation performance have the potential to benefit a large number of technologically critical areas.

As schematically illustrated in Fig. 1, the performance of a scintillator is related to a number of fundamental processes that involve the production, transport and interaction of carriers produced by the bombardment of those high-energy particles. At its most basic level, scintillation starts with the conversion of the high-energy incoming particle to a cascade of excited electrons and holes within the material. These hot carriers migrate through the material until, in many cases, they encounter a common recombination center, or activator, at which point they recombine, emitting visible or near-visible photons that are imaged with cameras.

In an ideal scenario, where the entire amount of incident energy is converted to photons in the visible or near-visible range of the spectrum and the energy lost as heat or to other non-radiative pathways is negligible, the number of photons that are produced would be the energy of that incoming particle, $E_{\gamma}$, divided by the band gap of the material $E_{b g}$. However, there are numerous factors that reduce the efficiency from this ideal case. First, the conversion process is not completely efficient. Many of the electrons and holes that are created have energies much higher than the band gap, reducing the total number that is produced. Then, as the carriers migrate through the material, they may get trapped at defects or impurities in the material. Further, they may recombine through non-radiative processes that do not lead to photon production. Finally, many activators commonly used in practice such as Ce and Eu have quantum efficiencies close to 1 and activator concentrations of about 1-5\% minimize excitation losses arising from energy transfer to the activator. However, even high concentrations of activator ions do not guarantee high efficiency of the transfer step, especially when the distances between thermalized electrons and holes are large in comparison with the radii of their recombination as well as with the distances between activator ions [8]. Thus, in all cases, the actual efficiency of the scintillator is significantly less than the ideal:

$n=\frac{E_{\gamma}}{\beta E_{b g}}<\frac{E_{\gamma}}{E_{b g}}$

where $n$ is the number of photons actually produced and $\beta$ is an empirical factor accounting for all of the losses. $\beta$ is almost always at least 2.5 , and in many cases is significantly larger. 
Each step can be targeted to improve the overall scintillator performance [37]. For example, to mitigate the effects of traps, either defect engineering [32], with the goal of eliminating the defects responsible for the traps, or band-edge engineering [7] to eliminate the effects of the traps, can improve transport processes. These kinds of efforts have improved the light output in garnets almost 5-fold [29, 31] and seem promising for perovskites as well [25]. However, even if all traps, or their effects, are eliminated, there is a chance that the carriers escape the local region and are thus unable to recombine and scintillate.

Recently, Belsky et al. [4] described a model in which a multicomponent compound, if the elements segregate in the right way, could limit the mean free path of carriers and thus enhance recombination. Specifically, they considered a case in which an $A$ $B$ two phase system phase separated into $A$-rich and $B$-rich domains, as schematically illustrated in Fig. 2(left panel). They showed that, within this model, if for example the $B$ domains had larger band gaps and thus band edges that were further from the Fermi level than the $A$ regions, those would act as barriers to transport, confining the carriers to the $A$ regions and thus enhancing their ability to find activators. Thus, by tuning the phase structure of the material, overall scintillator performance could be improved.

In this work, inspired by that conclusion, we consider the potential ability of Ruddlesden-Popper (RP) phases to reduce the mean free path of carriers and enhance recombination. RP phases are modular layered structures that arise in non-stoichiometric $A_{B O}$ type perovskites with the general formula $A_{n+1} B_{n} \mathrm{O}_{3 n+1} \quad(n=1,2,3)$. These can be viewed as extra planes of the rocksalt-structured $A \mathrm{O}$ phase interwoven throughout the $A B \mathrm{O}_{3}$ matrix. Thus, these are $A$ O-rich perovskites. Some experimentally observed examples of RP phases are shown in Fig. $3 \mathrm{a}, \mathrm{b}$. If these $A \mathrm{O}$ planes are arranged periodically, they produce unique diffraction peaks. However, often, they are arranged randomly, as we have seen in previous work on flash sintered $\mathrm{SrTiO}_{3}$ [18]. Because, in some general sense, these structures can be viewed as composites of $A B \mathrm{O}_{3}$ with interwoven $A O$ secondary "phases," we examine how well those $\mathrm{RP}$ faults (defined here as single planes of $A \mathrm{O}$ embedded in $A B \mathrm{O}_{3}$ ) might act as barriers for carrier transport, acting to confine them and enhance their

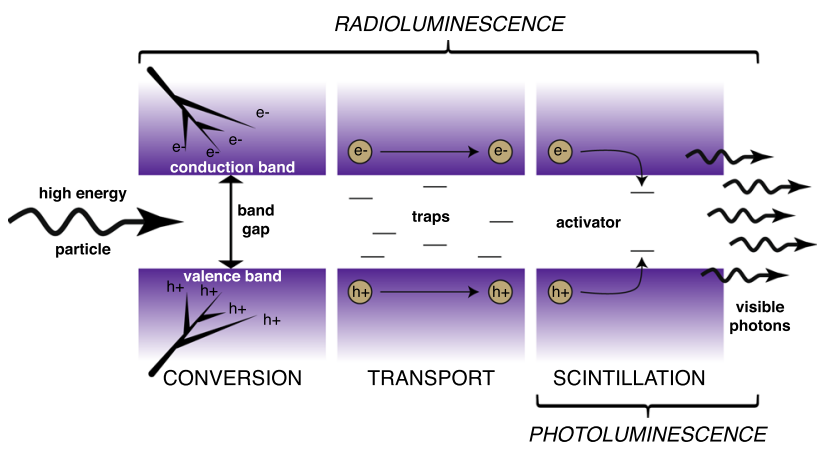

Figure 1 Schematic illustrating the basic processes involved during scintillation. First, high-energy particles are converted to hot carriers - electrons and holes-within the material. These carriers then migrate through the material, where they may be trapped at states induced by defects and impurities, reducing efficiency. Finally, some fraction of those carriers reach an activator site, often $\mathrm{Ce}^{3+}$ in many scintillators, at which point they recombine, emitting photons at energies suitable for visible detection.

ability to find an activator site for scintillation. The proposed geometry, schematically illustrated in Fig. 2(right panel), would be advantageous if it were able to constrain carriers in a similar way, as all regions would be confining, instead of just isolated islands of $\mathrm{A}$.

To that end, in this work we use density functional theory (DFT) to examine how the band structure changes near RP faults to identify whether the RP faults might act as barriers to electron and/or hole transport. We then use ab initio molecular dynamics (AIMD) to explicitly simulate the structural dynamics of bulk as well as RP phase structures at ambient conditions. Using non-adiabatic molecular dynamics (NAMD), we further investigate the impact of these structural fluctuations on the carrier dynamics in model structures to demonstrate the potential reduction in carrier mean free path and thus the enhancement in potential scintillation. However, we also see that non-radiative recombination is also increased by the presence of the interfaces at RP phase. As this work is meant as a proof-of-principle, we focus our efforts on the non-scintillating perovskite $\mathrm{SrTiO}_{3}$ for convenience, though we expect the general conclusions should hold for other chemistries. 


\section{Methodology}

The total energy calculations were performed using the Vienna ab initio simulation package (VASP) [21, 22] implementation of the DFT [20] framework. The generalized gradient approximation (GGA) [35] exchange correlation functional is used in the form of the parameterization proposed by Perdew, Burke, and Ernzerhof (PBE) [36]. Brillouin zone integrations were performed using a $\Gamma$-point mesh with at least $5000 k$-points per reciprocal atom. Full relaxations were carried out using the MethfesselPaxton smearing method [28] of order one and a final self-consistent static calculation with the tetrahedron smearing method with Blöchl corrections [5]. A plane wave cutoff energy of $500 \mathrm{eV}$ was used and spin polarization was accounted for in all the calculations. All relaxations were carried out until changes in the total energy between relaxation steps were within $1 \times$ $10^{-6} \mathrm{eV}$ and atomic forces on each of the atoms were smaller than $0.01 \mathrm{eV} / \AA$.

The site-projected and orbital-projected density of states (DOS) were calculated by projecting the wave functions onto atom-centered spherical harmonics for each atom. The corresponding DOS for each structural unit was estimated by summing the site-projected and orbital-projected DOS for the atoms comprising the structural unit. The electronic structure module of the pymatgen [33] software package was used to extract this data.

To study the charge carrier recombination at ambient conditions, we further use mixed quantumclassical NAMD simulations with the decoherenceinduced surface hopping (DISH) methodology $[6,13,16]$. We consider electrons and nuclei as quantum mechanical and semiclassical particles, respectively. The method has been previously used by one of the authors and several others to explore carrier recombination in semiconductors $[9,11,19,24,27]$. To perform AIMD simulations, we construct supercells containing 192 and 135 atoms for RP phase and bulk phase $\mathrm{SrTiO}_{3}$, respectively. For these simulations, we use the same 1D geometry that is illustrated in Fig. 3c. A plane-wave energy cutoff of $400 \mathrm{eV}$, PBE-GGA exchange-correlation functions, a Gamma-point mesh and a MD time step of $1 \mathrm{fs}$ have been used for all the AIMD simulations.

Considering the $0 \mathrm{~K}$ DFT optimized structures as the starting geometry for AIMD simulations, we

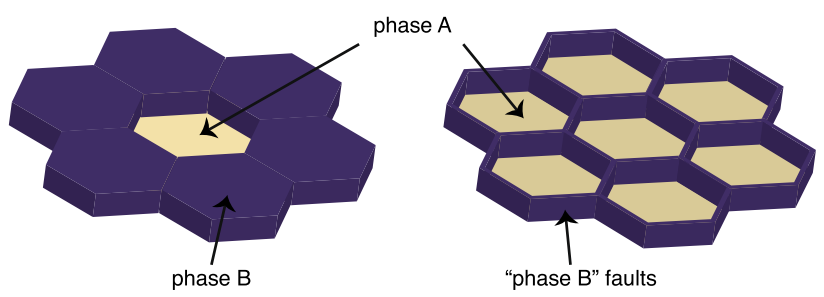

Figure 2 Schematic of how the phase structure might be used to constrain carrier dynamics to enhance scintillation. (left panel) The concept as proposed by Belsky et al. [4], in which phase A has a smaller band gap than phase B and thus acts as a local trap or confining region for carriers. (right panel) The concept explored here, in which "phase B," the phase with the larger band gap, is a network of RP faults.

simulated heating of the system up to $300 \mathrm{~K}$ using repeated velocity rescaling for 4 ps. Following that, the trajectories for another 3 ps were simulated within the canonical ensemble (NVT), ensuring thermal equilibrium. Finally, we generated trajectories for $10 \mathrm{ps}$ within the microcanonical ensemble (NVE) and used the last 4 ps of it to perform NAMD simulations.

All 4000 geometries along the AIMD trajectories and 1000 stochastic realizations of the DISH process for each geometry were used to evaluate the electronhole recombination as implemented in the PYXAID code [1, 2]. For photo-excitation and subsequent carrier relaxation, only the band-edge states are primarily considered as the involved electronic states. To model the initial band-to-band excitation of the material, we promote a single electron from the Kohn-Sham (KS) state of the valence band edge to the KS state of the conduction band edge. Thus, essentially, the simulation uses an active space where a subset of complete KS-states is considered to construct the basis configuration. A detailed description of the methodology can be found in the paper by Akimov et al. [1, 2]. Note that high-energy excitations that require the involvement of the electronic states much deeper in the bands have not been considered in the current study. We further investigate the puredephasing process by considering the optical-response theory and evaluate the decoherence time for these materials [14]. The decoherence time can be calculated by fitting a single-Gaussian function with the form [41],

$f(t)=\exp \left(-(t / \tau)^{2} / 2\right)$.

The short-time linear approximation has been used to 

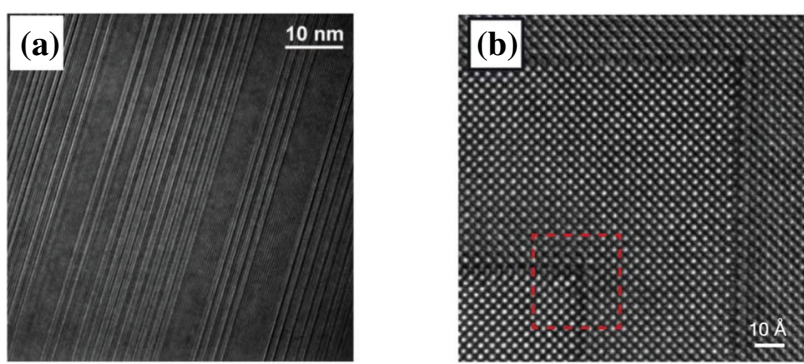

(c)
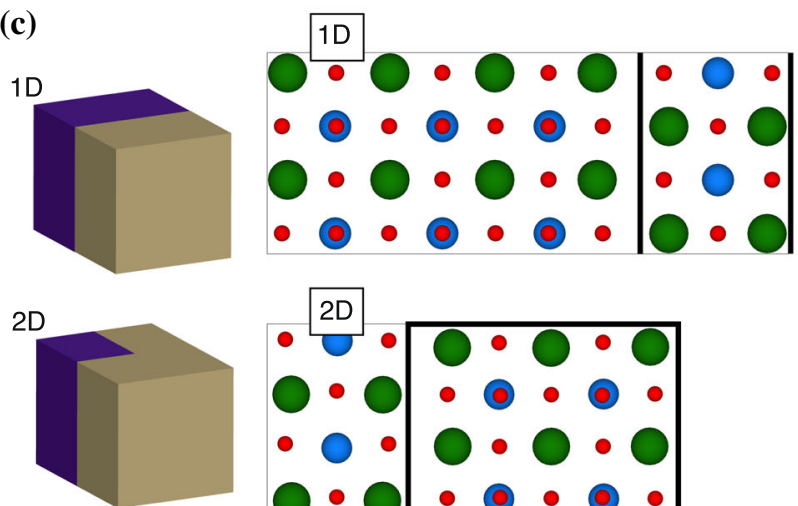

$3 \mathrm{D}$
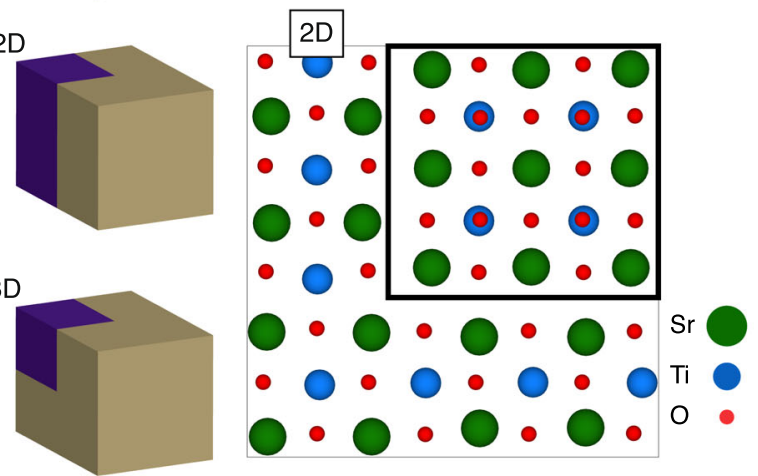

Figure 3 High-resolution transmission electronic microscopy images showing presence of 1D (a) and 2D (b) RuddlesdenPopper phases imaged in previous studies. Reproduced with permissions from $[18,39]$. c Schematic of the types of faulted structures considered in this work. As the dimensionality of the faulted system is increased, new faults are added in orthogonal directions. The structures for the 1D and 2D faulted systems actually considered in the DFT calculations are also shown, with the positions of the faults highlighted by the thick black lines.

fit the exponential increase of the non-radiatively recombined charge population in the ground state of the systems [30]. This further allowed us to calculate the electron-hole non-radiative recombination time across the electronic band gap. The recombination time $(\tau)$ was evaluated by fitting the change in population to the following equation [10, 30],

$g(t)=1-\exp (-t / \tau)$.

Note that we did not perform computationally expensive NAMD simulations that account for the spin-orbit coupling (SOC) effects, which can change the recombination rates at a quantitative level [23]. However, recent studies indicate that NAMD without SOC effects can successfully capture the experimental trend of the non-radiative recombination rates in perovskites materials [11, 12]. We also did not model the possibility of having explicit small polaron formation in these oxide materials [15].

\section{Results and analysis}

Using DFT, we considered how RP faults arranged in 1, 2 and 3 dimensions impacted the electronic structure of the system. While RP phases have orderly arrangements of the faults, generally the faults can lie in any dimension, forming a complex three-dimensional network of $A \mathrm{O}$-like inclusions. Given that $\mathrm{SrO}$ has a much larger calculated band gap than $\mathrm{SrTiO}_{3}$, (3.27 vs $1.79 \mathrm{eV}$, respectively), one might expect that the faults act as regions of locally larger band gap and potentially barriers for electron and hole transport. Figure $3 c$ provides both a schematic representation of the different structures that might form as RP faults are arranged in different ways as well as actual structures used in the DFT calculations to determine their effects. The positions of the faults are highlighted. In the case of the 1D structure, we chose an arrangement where the spacing between the two faults is not equal in each direction. This allows us to understand how the spacing between faults impacts the electronic structure of the material.

Panels in Fig. 4c provide the orbital-projected DOS as determined for the structural units highlighted in Fig. $4 \mathrm{a}$, while Fig. $4 \mathrm{~b}$ shows the spatially varying band gap normal to the fault direction on a finer scale.

The site projection clearly reveals a significant variation of the band gap and other aspects of the electronic structure with distance from the RP faults in the system. While overall the band gap of the structure is reduced compared to perfect $\mathrm{SrTiO}_{3}(1.57$ vs $1.79 \mathrm{eV}$, respectively), there are regions where locally the band gap increases to over $2 \mathrm{eV}$, a consequence of the higher band gap of the extra rocksaltstructured faults. Correspondingly, the higher band gap lies exactly where the extra plane of $\mathrm{SrO}$ is introduced into the material. Thus, as hypothesized, the RP faults do act, to some degree, like the inclusion of a second phase, at least in terms of the band structure. We note that the values for the site-projected DOS and band gap depend on exactly how space is partitioned, and that is the reason that 
(b)
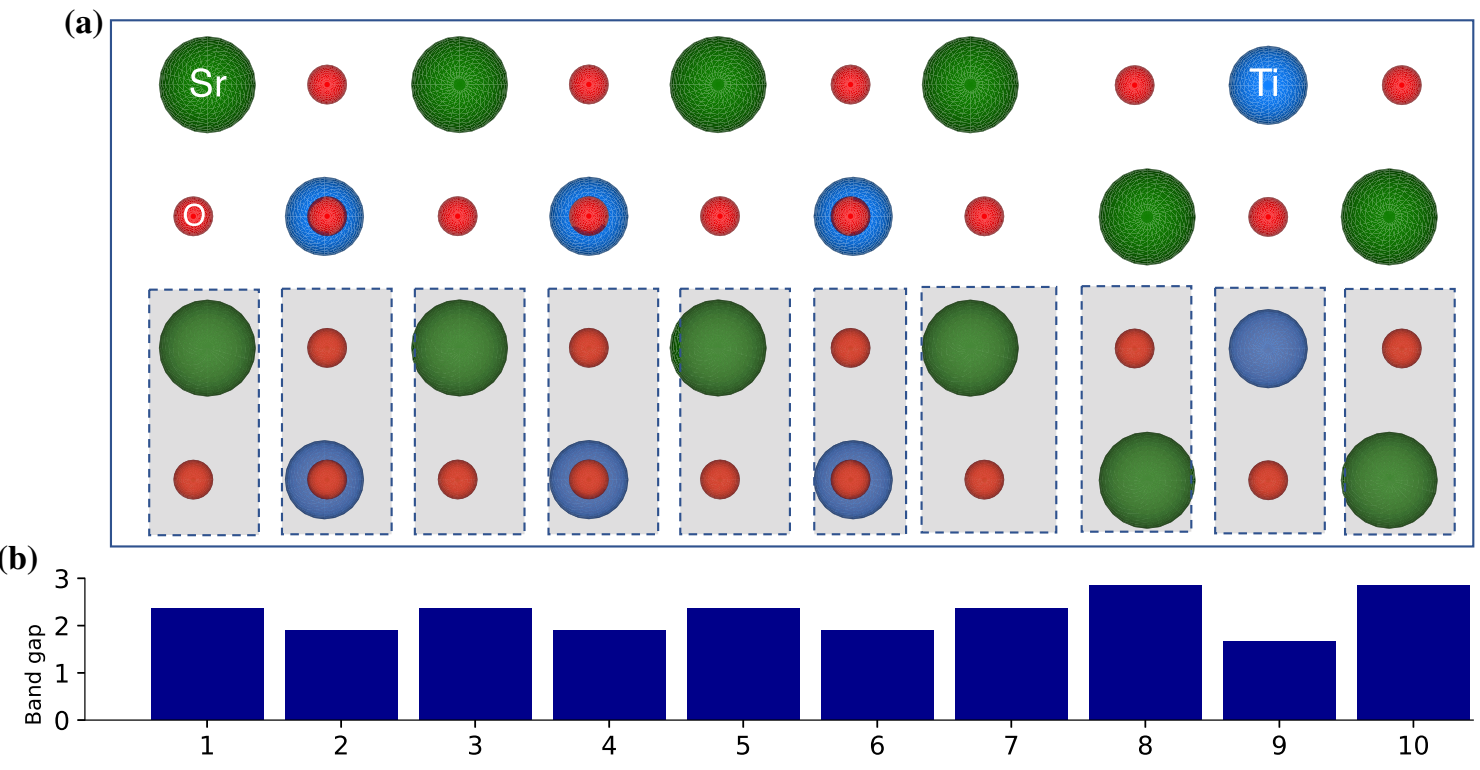

(c)

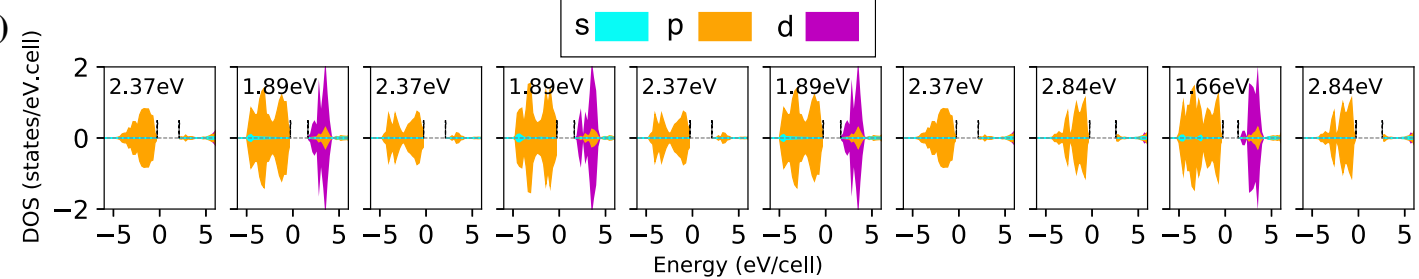

Figure 4 Results of DFT calculations on the 1D faulted structure shown in Fig. 3c. a The structure of the 1D faulted system with the structural units upon which the spatially varying band gaps in

Fig. $4 \mathrm{~b}, \mathrm{c}$ do not completely agree on the local values of the band gaps, but the general trend-that the band gap is higher near the RP faults-is the same in either case.

It is interesting to note that the effect is very short ranged. Only two planes away from the faulted atoms, the band gap behaves just as in bulk $\mathrm{SrTiO}_{3}$. Thus, the inclusion of the RP faults does not perturb the electronic structure of the matrix material to any appreciable extent. The one layer of $\mathrm{TiO}_{2}$ between the two faults in the shorter direction has a band gap that is very similar to the band gap of the same type of structural unit in the bulk, again highlighting the very short-ranged nature of the perturbation of the faults on the electronic structure. Thus, the faults act like a very sharp barrier to carriers that might be present in the matrix.

The orbital-projected DOS is presented in Fig. 4c. These show that the band gap effectively is the gap between the lower edge of the $p$ orbital bands and the upper edge of the $d$ orbital bands, belonging primarily to the $\mathrm{O}$ and $\mathrm{Ti}$ ions, respectively. The $\mathbf{b}$ and orbital-projected DOS in $\mathbf{c}$ are determined. $\mathbf{b}$ The band gap as a function of position in the direction normal to the fault plane. c Orbital-projected DOS as a function of position.

difference in band gap across structural units is mainly due to a upward shift in the $d$ orbital bands while the position of the upper edge of the $p$ orbital bands remain almost constant over all the structural units.

Similar results are obtained for the 2D and 3D structures. For the sake of brevity and clarity, we only show the results for the 2D structure in Fig. 5. Interestingly, for the 2D and 3D structures, the overall band gap is increased to over $2 \mathrm{eV}(2.29$ and $2.12 \mathrm{eV}$, respectively). The site-projected band gap has increased everywhere, but the band gap at the faults is still significantly higher than in the rest of the material. The details of this increase depend on precisely how the DOS is projected onto structural units in the material, but the basic behavior is similar regardless of the details. Importantly, as highlighted in Fig. 5, the gap at the corner where the two orthogonal faults meet is also high, meaning there is no "hole" in the band structure where carriers might slip through. This is also true in the 3D structure, where three faults come to a point: even in this case, 

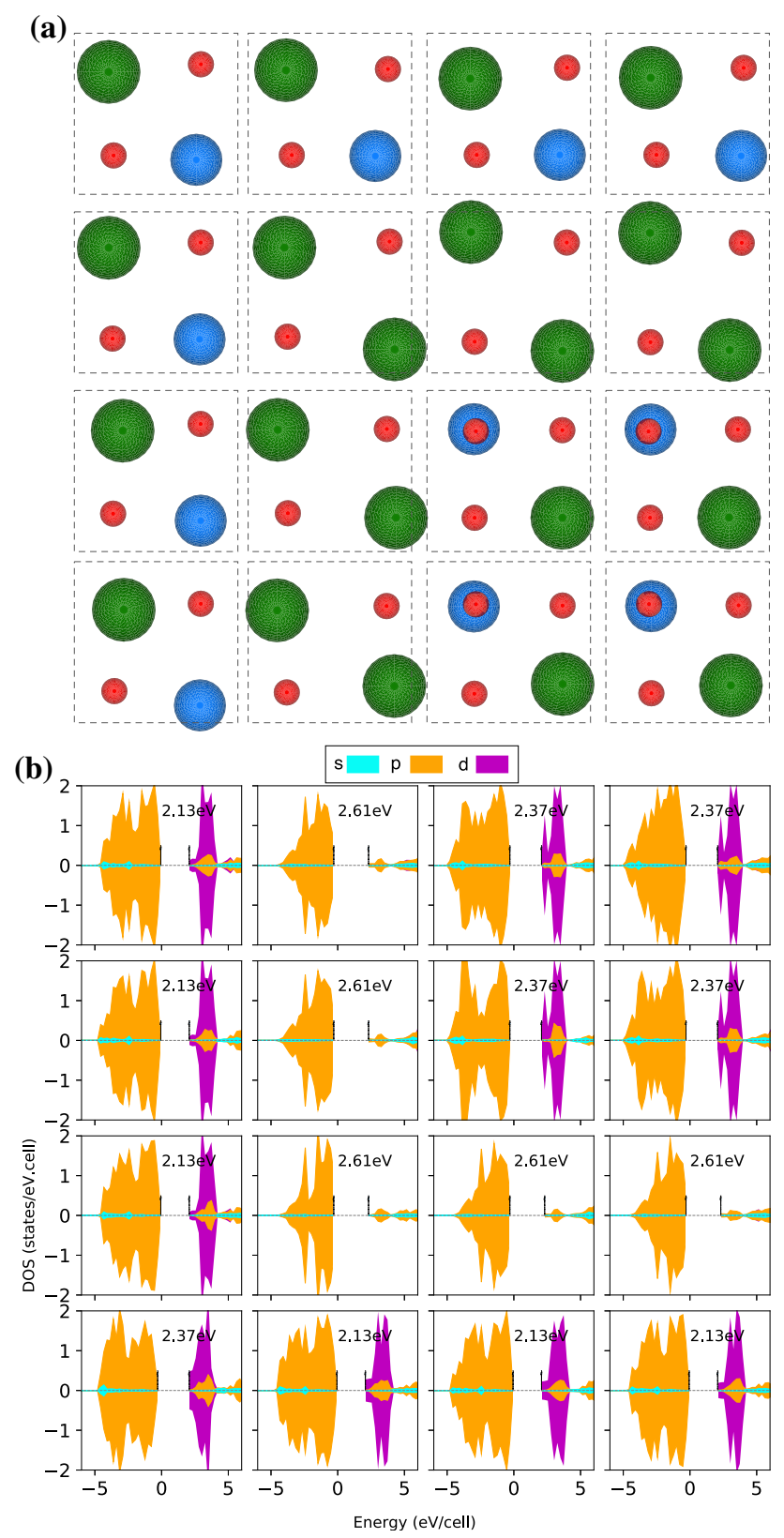

Figure 5 Results of DFT calculations on the 2D faulted structure shown in Fig. 3c. a The structure of the 2D faulted system with the structural units upon which the orbital-projected DOS in $\mathbf{b}$ are determined highlighted (purple boxes). $\mathbf{b}$ The orbital-projected DOS as a function of position.

the site-projected band gap along the entire surface of the faulted region is higher than the neighboring bulk.

The hypothesis for examining the effect of RP faults on the electronic structure in $\mathrm{SrTiO}_{3}$ was based on the fact that $\mathrm{SrO}$ has a larger band gap than $\mathrm{SrTiO}_{3}$ and the faults might present a barrier to carrier transport. However, as noted, $\mathrm{SrTiO}_{3}$ is not a scintillating perovskite. Thus, we also examined the behavior of RP faults in $\mathrm{SrHfO}_{3}$, which is known to be scintillating [26], see Fig. 6. This is an interesting contrast, as $\mathrm{SrHfO}_{3}$ has a larger band gap than SrO: 3.73 versus $3.27 \mathrm{eV}$. Based on the original hypothesis, we might expect that the site-projected band gaps are lower at the faults. However, DFT calculations reveal this not to be the case. While the overall band gap of the material in a 1D faulted geometry, $3.72 \mathrm{eV}$, is indeed much higher than for $\mathrm{SrTiO}_{3}$, the faulted region exhibits a projected band gap of $4.51 \mathrm{eV}$. Thus, even in this case, the faults act as a potential barrier of transport and provide support that these effects may exist in a scintillating perovskite.

While the DFT calculations suggest that the electronic structure associated with the RP faults in nonstoichiometric perovskites is changed such that the faults might act as barriers to carriers, we use AIMD and NAMD to explicitly examine the role that these faults have on the properties of carriers. For these simulations, we use the same 1D geometry that is illustrated in Fig. 3c.

The key results are shown in Fig. 7. Figure 7a highlights the impact that the faults have on the recombination rate of electrons and holes. Only the non-radiative recombination rate is calculated as the simulations do not include a mechanism for radiative recombination process. Regardless, the rate is directly

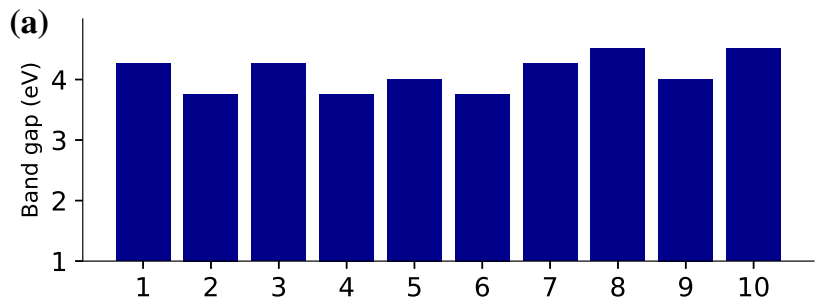

(b)

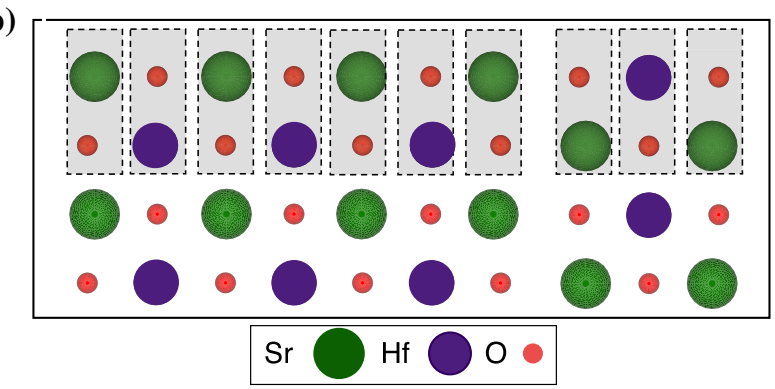

Figure 6 Results of DFT calculations on the 1D faulted structure for $\mathrm{SrHfO}_{3}$. a The band gap as a function of position in the direction normal to the fault plane. $\mathbf{b}$ The structure of the 1D faulted system with the defined structural units. 

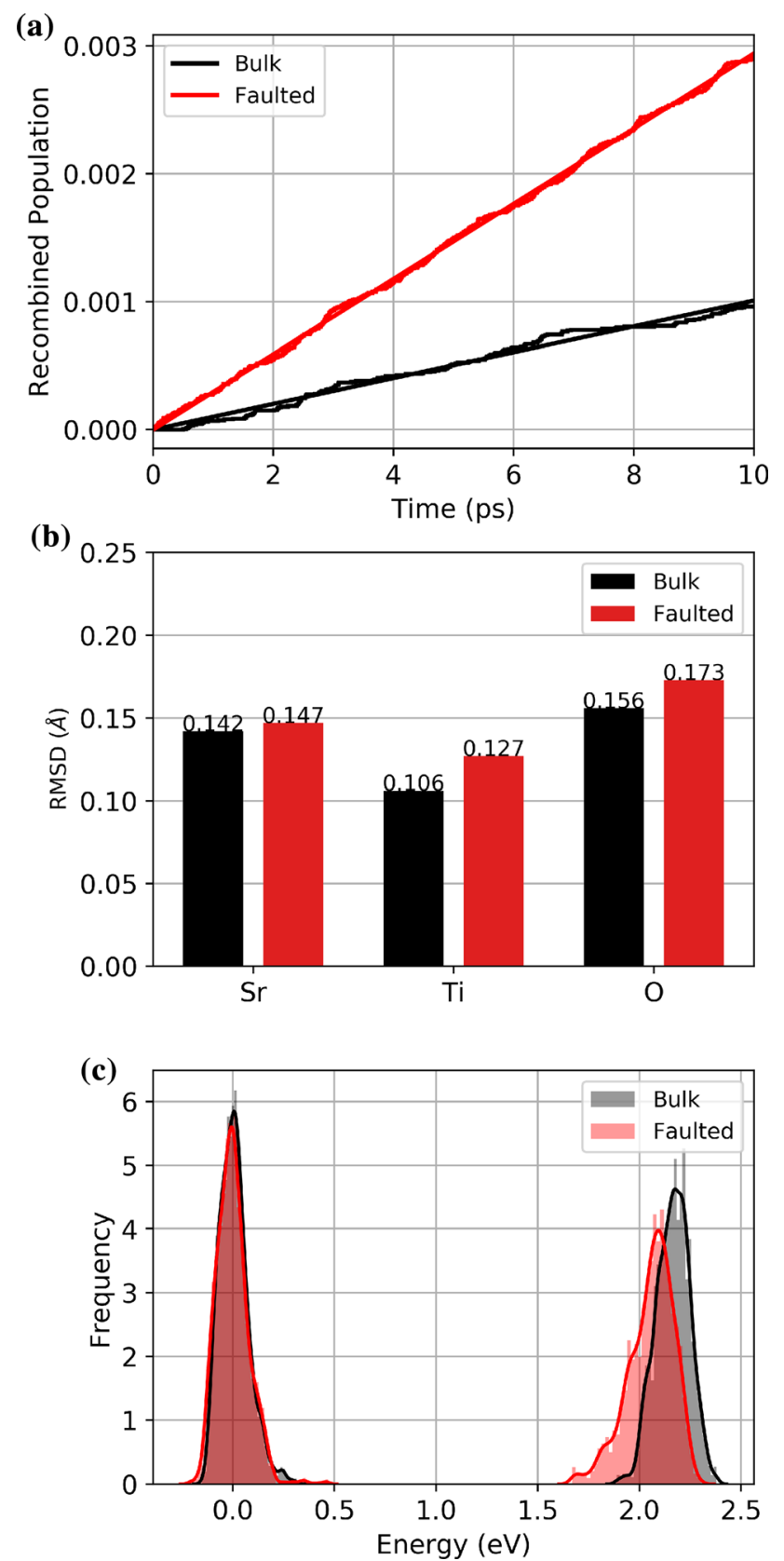

Figure 7 Results from the AIMD and NAMD simulations for the 1D faulted and bulk structures. a The population of recombined electrons and holes versus time in the two structures. $\mathbf{b}$ Magnitude of the vibrations of the different elements within both the perfect bulk and faulted structures over the course of the AIMD simulations. c Variation in the valence and conduction band edge state positions (scaled to most probable position of VBM as 0 $\mathrm{eV}$ ) in both the bulk and faulted system over the course of the AIMD simulations.

related to the mean free path of carriers within the system. As seen in Fig. 7a, the rate of non-radiative recombination is much higher in the faulted system than in the bulk system. Applying the short-time linear approximation of the exponentially increasing function (see Sect. 2), the lifetime of carriers is much smaller: $3.3 \mathrm{~ns}$ versus $9.9 \mathrm{~ns}$ in the faulted versus bulk structures, respectively. This suggests that the carriers in the faulted structure have a shorter mean free path and thus, if radiative recombination mechanisms were included, such mechanisms might be enhanced. However, more clearly, non-radiative recombination, due to electron-phonon coupling, is certainly enhanced. This indicates that, if the faults do impart any benefit by confining carriers and enhancing their recombination, they do it at the expense of also increasing non-radiative loss.

Figure $7 \mathrm{~b}$ shows the root mean square displacement (RMSD) for individual atomic species present in the material. The RMSD describes the magnitude of thermal fluctuations of the species in both the bulk and faulted regions. All elements exhibit larger vibrations in the faulted system compared to the bulk system. This is particularly pronounced for $\mathrm{Ti}$ atoms near the fault as compared to $\mathrm{Ti}$ atoms away from the fault (not shown). This is indicative of the fact that inclusion of fault in the bulk makes the solid more dynamically active in general.

The electronic and lattice degrees of freedom dynamically couple at finite temperature, influencing the non-radiative charge carrier relaxation processes that significantly impact the charge carrier lifetime in semiconductors. Electron-phonon interactions as well as energy gaps predominantly determine the non-radiative carrier recombination rate in semiconductors. Thus, we further explore these factors in detail to understand the trend of carrier lifetime as shown in Fig. 7a.

The electronic and vibrational subsystems exchange energy through an inelastic electron-phonon scattering process at ambient conditions. Due to this, the excited electron dissipates its energy to the phonon modes, activating non-radiative recombination. The averaged non-adiabatic coupling (NAC) constants over complete trajectories quantify the strength of this inelastic electron-phonon scattering process. The higher the value of the NAC, the stronger the coupling between electrical and lattices degrees of freedom. This underpins the extent of the inelastic scattering process and further indicates faster charge recombination in the semiconductor. We find averaged NAC values for bulk and RP-faulted $\mathrm{SrTiO}_{3}$ are $19 \mathrm{meV}$ and $25 \mathrm{meV}$, respectively. The 
larger NAC in the faulted structure indicates faster non-radiative recombination, as we find in Fig. 7a.

Further, these thermal excursions also make the electronic structure very dynamic at ambient conditions. Figure $7 \mathrm{c}$ shows the variation in valence and conduction band edge state positions of the entire structure over the course of the MD simulation for both the 1D RP faulted structure and a perfect bulk reference structure. On average, as discussed earlier, the band gap of the faulted structure is lower than that of the bulk reference structure, more so than as determined from the static DFT calculations, which is presumably a consequence of the thermal fluctuations that distort the structure. Further, the variation is confined to the conduction band, which is comprised of Ti $3 \mathrm{~d}$ states and is more susceptible to the thermal fluctuations of Ti. The faulted structure also exhibits a wider variation in the band gap during the course of the AIMD simulation. This is a consequence of greater dynamical activity of the atoms within the faulted structure-the atoms in the faulted structure exhibit greater RMSD than do those in the bulk structure. Thus, not only do the thermal vibrations increase near a RP fault, but those vibrations induce a wider variation in the electronic structure of the material, also facilitating non-radiative recombination.

Taken together, these results indicate that, while $\mathrm{RP}$ faults may have the potential to act as barriers to electrons and holes, effectively corralling them and preventing their mutual escape, other effects can arise that may counter any benefit the confining potential offers. In particular, non-radiative processes may be enhanced that reduce the overall scintillating efficiency of the system.

\section{Discussion}

As proposed by Belsky et al. [4], a multi-phase composite structure can, if it has the appropriate electronic structure, enhance carrier recombination by reducing the mean free path of carriers. Essentially, carriers in one phase are confined and thus recombine faster. We have shown that the RP faults that are common in non-stoichiometric perovskites can potentially enhance radiative recombination. Thus, at least for applications in which high recombination rates are beneficial, such as scintillation, non-stoichiometry may offer one route to achieve higher rates.

It is interesting to note that similar considerations apply to other defect recombination problems. In particular, in previous work we developed a model of interstitial and vacancy recombination in the context of radiation damage in composite structures $[34,38]$. There, we found that if the defect energetics were such that both interstitials and vacancies preferred the same phase, recombination would be enhanced, much like the behavior of electrons and holes in the current model.

Of course, in real applications, other factors must be considered. For example, the RP faults may prove to be sites for activator dopant segregation, perturbing the ability of carriers to find them. RP faults in $\mathrm{SrTiO}_{3}$ are known to attract point defects [39] and very well could attract dopants. However, the ability to induce a high density of RP faults in perovskites via methods such as flash sintering [18] does provide a reasonably easy route to modify the material structure to enhance scintillator efficiency. Tuning the flash sintering parameters (voltage and temperature ramp) could enable precise control of the density of these faults. Techniques such as conventional and laser molecular beam epitaxy (MBE) [17, 40], which enable control of the synthesis of matter at the atomic scale, have been demonstrated to be very useful in the realization of atomically sharp artificial heterostructures to control the spacing and density of planar defects in perovskites, garnets and rutile structures.

One critical result of this work is that, while the RP faults may act as barriers to carrier migration and thus potentially enhance radiative recombination, this comes at a price. The larger atomic flexibility at the interface leads to greater vibrational degrees of freedom, also enhancing electron-phonon coupling and strengthening non-adiabatic coupling between band edge states. These dynamical effects further result in faster non-radiative recombination in the faulted structure compared to the bulk phase. This is likely a feature of most interfaces and is an aspect that Belsky et al. [4] did not include in their model. Thus, any interfaces would lead to deleterious effects that may compete with the enhanced radiative recombination that might arise from the multi-phase structure. At this time, we do not know which would dominate in faulted $\mathrm{SrTiO}_{3}$. That said, there is an opportunity for materials design here. The enhanced 
electron-phonon coupling observed here could be tuned by the appropriate choice of chemistry. That is, by choosing a different perovskite chemistry, it is possible this could be reduced, by choosing B cations that are more massive than Ti. On the other hand, the barrier effect of the faults, as long as the local band gap is larger, would be largely unaffected. Thus, we can speculate that the balance between enhanced radiative versus non-radiative recombination can be at least partially tuned by appropriate choices in chemistry.

\section{Conclusion}

We used $\mathrm{SrTiO}_{3}$ as a representative structure to investigate the potential ability of RP phases to reduce the mean free path of carriers and enhance recombination. The problem was approached from three aspects: (1) using DFT to identify RP fault induced band structure changes in the vicinity of the fault which may act as barriers to electron and/or hole transport, (2) explicit simulation of the structural dynamics at ambient conditions using AIMD and (3) investigation of the role of carrier dynamics (via reduction of carrier mean free path) in the enhancement of scintillation using NAMD. DFT calculations indicated that the electronic structure associated with the RP faults in non-stoichiometric perovskites is sufficiently altered so that the faults might act as barriers to carriers. Molecular dynamics simulations indicated faster non-radiative recombination for the RP phase which may reduce the overall scintillating efficiency of the system. Thus, introduction of RP faults in perovskite chemistries may give rise to competing effects of increasing desired radiative recombination rates accompanied by an unwanted increase in rates of non-radiative recombination. This scenario, however, affords the possibility of tuning of the effective scintillation efficiency by varying the perovskite chemistry and by appropriate arrangements of RP faults in the bulk material. In principle, the results discussed in this work may be extended to any other network of planar defects, such as grain boundaries, phase boundaries or stacking faults which afford the opportunity to manipulate the local electronic structure. Especially in the case of nanocrystalline perovskite scintillators [3], carefully engineered defect states may give rise to numerous technological applications arising from the altered optical properties. Thus, any morphology that creates a potential well, and enables tuning of the local electronic structure could potentially facilitate the improvement of the overall scintillator efficiency.

\section{Acknowledgements}

Research presented in this article was supported by the Laboratory Directed Research and Development program of Los Alamos National Laboratory under project numbers 20190656PRD4 and 20190043DR. Los Alamos National Laboratory is operated by Triad National Security, LLC, for the National Nuclear Security Administration of US Department of Energy (Contract No. 89233218CNA000001). This work was done in part at the Center for Nonlinear Studies (CNLS), Los Alamos National Laboratory. Computational support for this work was provided by LANLs high-performance computing clusters.

\section{Declarations}

Conflict of interest The authors declare that they have no conflict of interest.

Open Access This article is licensed under a Creative Commons Attribution 4.0 International License, which permits use, sharing, adaptation, distribution and reproduction in any medium or format, as long as you give appropriate credit to the original author(s) and the source, provide a link to the Creative Commons licence, and indicate if changes were made. The images or other third party material in this article are included in the article's Creative Commons licence, unless indicated otherwise in a credit line to the material. If material is not included in the article's Creative Commons licence and your intended use is not permitted by statutory regulation or exceeds the permitted use, you will need to obtain permission directly from the copyright holder. To view a copy of this licence, visit http://creativecommons.org/licen ses/by $/ 4.0 /$.

\section{References}

[1] Akimov AV, Prezhdo OV (2013) The PYXAID program for non-adiabatic molecular dynamics in condensed matter systems. J Chem Theory Comput 9(11):4959-4972 
[2] Akimov AV, Prezhdo OV (2014) Advanced capabilities of the PYXAID program: integration schemes, decoherence effects, multiexcitonic states, and field-matter interaction. J Chem Theory Comput 10(2):789-804

[3] Becker MA, Vaxenburg R, Nedelcu G, Sercel PC, Shabaev A, Mehl MJ, Michopoulos JG, Lambrakos SG, Bernstein N, Lyons JL et al (2018) Bright triplet excitons in caesium lead halide perovskites. Nature 553(7687):189-193

[4] Belsky A, Gektin A, Vasil'ev AN (2019) Influence of disorder in scintillating solid solutions on thermalization and recombination of electronic excitations. Physica Status Solidi (b) 1900535

[5] Blöchl PE, Jepsen O, Andersen OK (1994) Improved tetrahedron method for Brillouin-zone integrations. Phys Rev B 49(23): 16223

[6] Craig CF, Duncan WR, Prezhdo OV (2005) Trajectory surface hopping in the time-dependent Kohn-Sham approach for electron-nuclear dynamics. Phys Rev Lett 95(16):163001

[7] Fasoli M, Vedda A, Nikl M, Jiang C, Uberuaga B, Andersson D, McClellan K, Stanek C (2011) Band-gap engineering for removing shallow traps in rare-earth $\mathrm{Lu}_{3} \mathrm{Al}_{5} \mathrm{O}_{1} 2$ garnet scintillators using $\mathrm{Ga}^{3+}$ doping. Phys Rev B 84(8):081102

[8] Gektin AV, Belsky AN, Vasil'ev AN (2013) Scintillation efficiency improvement by mixed crystal use. IEEE Trans Nucl Sci 61(1):262-270

[9] Ghosh D, Acharya D, Zhou L, Nie W, Prezhdo OV, Tretiak S, Neukirch AJ (2019) Lattice expansion in hybrid perovskites: effect on optoelectronic properties and charge carrier dynamics. J Phys Chem Lett 10(17):5000-5007

[10] Ghosh D, Acharya D, Pedesseau L, Katan C, Even J, Tretiak S, Neukirch AJ (2020) Charge carrier dynamics in two-dimensional hybrid perovskites: Dion-Jacobson vs Ruddlesden-Popper phases. J Mater Chem A 8(42):22009-22022

[11] Ghosh D, Neukirch AJ, Tretiak S (2020) Optoelectronic properties of two-dimensional bromide perovskites: influences of spacer cations. J Phys Chem Lett 11(8):2955-2964

[12] Gong X, Voznyy O, Jain A, Liu W, Sabatini R, Piontkowski Z, Walters G, Bappi G, Nokhrin S, Bushuyev O et al (2018) Electron-phonon interaction in efficient perovskite blue emitters. Nat Mater 17(6):550-556

[13] Granucci G, Persico M, Zoccante A (2010) Including quantum decoherence in surface hopping. J Chem Phys 133(13):134111

[14] Hamm P (2005) Principles of nonlinear optical spectroscopy: a practical approach. Univ Zurich 41(5):77

[15] Husanu MA, Vistoli L, Verdi C, Sander A, Garcia V, Rault J, Bisti F, Lev LL, Schmitt T, Giustino F et al (2020) Electronpolaron dichotomy of charge carriers in perovskite oxides. Commun Phys 3(1):1-8
[16] Jaeger HM, Fischer S, Prezhdo OV (2012) Decoherenceinduced surface hopping. J Chem Phys 137(22):22A545

[17] Kanai M, Kawai T, Kawai S (1991) Atomic layer and unit cell layer growth of (ca, sr) cuo2 thin film by laser molecular beam epitaxy. Appl Phys Lett 58(7):771-773

[18] Karakuscu A, Cologna M, Yarotski D, Won J, Francis JS, Raj R, Uberuaga BP (2012) Defect structure of flash-sintered strontium Titanate. J Am Ceram Soc 95(8):2531-2536

[19] Kilina S, Velizhanin KA, Ivanov S, Prezhdo OV, Tretiak S (2012) Surface ligands increase photoexcitation relaxation rates in CDSE quantum dots. ACS Nano 6(7):6515-6524

[20] Kohn W, Sham LJ (1965) Self-consistent equations including exchange and correlation effects. Phys Rev 140(4A):A1133

[21] Kresse G, Furthmüller J (1996) Efficiency of ab-initio total energy calculations for metals and semiconductors using a plane-wave basis set. Comput Mater Sci 6(1):15-50

[22] Kresse G, Furthmüller J (1996) Efficient iterative schemes for ab initio total-energy calculations using a plane-wave basis set. Phys Rev B 54(16):11169

[23] Li W, Zhou L, Prezhdo OV, Akimov AV (2018) Spin-orbit interactions greatly accelerate nonradiative dynamics in lead halide perovskites. ACS Energy Lett 3(9):2159-2166

[24] Liu J, Kilina SV, Tretiak S, Prezhdo OV (2015) Ligands slow down pure-dephasing in semiconductor quantum dots. ACS Nano 9(9):9106-9116

[25] Liu XY, Pilania G, Talapatra AA, Stanek CR, Uberuaga BP (2020) Band-edge engineering to eliminate radiation-induced defect states in perovskite scintillators. ACS Appl Mater Interfaces 12(41):46296-46305

[26] van Loef EV, Higgins WM, Glodo J, Brecher C, Lempicki A, Venkataramani V, Moses WW, Derenzo SE, Shah KS (2007) Scintillation properties of $\mathrm{SrHfo}_{3}: \mathrm{Ce}^{3+}$ and $\mathrm{BaHfo}_{3}: \mathrm{ce}^{3+}$ ceramics. IEEE Trans Nucl Sci 54(3):741-743

[27] Long R, Liu J, Prezhdo OV (2016) Unravelling the effects of grain boundary and chemical doping on electron-hole recombination in ch3nh3pbi3 perovskite by time-domain atomistic simulation. J Am Chem Soc 138(11):3884-3890

[28] Methfessel M, Paxton A (1989) High-precision sampling for Brillouin-zone integration in metals. Phys Rev B 40(6):3616

[29] Moszyński M, Ludziejewski T, Wolski D, Klamra W, Norlin L (1994) Properties of the YAG: Ce scintillator. Nucl Instrum Methods Phys Res Sect A 345(3):461-467

[30] Nijamudheen A, Akimov AV (2017) Criticality of symmetry in rational design of chalcogenide perovskites. J Phys Chem Lett 9(1):248-257

[31] Nikl M, Yoshikawa A, Kamada K, Nejezchleb K, Stanek C, Mares J, Blazek K (2013) Development of LuAG-based scintillator crystals - a review. Prog Cryst Growth Charact Mater 59(2):47-72 
[32] Nikl M, Kamada K, Babin V, Pejchal J, Pilarova K, Mihokova E, Beitlerova A, Bartosiewicz K, Kurosawa S, Yoshikawa A (2014) Defect engineering in Ce-doped aluminum garnet single crystal scintillators. Crystal Growth Des 14(9):4827-4833

[33] Ong SP, Richards WD, Jain A, Hautier G, Kocher M, Cholia S, Gunter D, Chevrier VL, Persson KA, Ceder G (2013) Python materials genomics (pymatgen): a robust, opensource python library for materials analysis. Comput Mater Sci 68:314-319

[34] Pedro Uberuaga B, Martinez E, Bi Z, Zhuo M, Jia Q, Nastasi M, Misra A, Caro A (2013) Defect distributions and transport in nanocomposites: a theoretical perspective. Mater Res Lett 1(4):193-199

[35] Perdew JP, Wang Y (1992) Accurate and simple analytic representation of the electron-gas correlation energy. Phys Rev B 45(23):13244

[36] Perdew JP, Burke K, Ernzerhof M (1996) Generalized gradient approximation made simple. Phys Rev Lett 77(18):3865
[37] Rodnyi PA (1997) Physical processes in inorganic scintillators, vol 14. CRC Press, Boca Raton

[38] Uberuaga BP, Choudhury S, Caro A (2015) Ideal sinks are not always ideal: radiation damage accumulation in nanocomposites. J Nucl Mater 462:402-408

[39] Won J, Vernon LJ, Karakuscu A, Dickerson RM, Cologna M, Raj R, Wang Y, Yoo SJ, Lee SH, Misra A et al (2013) The role of non-stoichiometric defects in radiation damage evolution of $\mathrm{SrTiO}_{3}$. J Mater Chem A 1(32):9235-9245

[40] Yang G, Lu H, Chen F, Zhao T, Chen Z (2001) Laser molecular beam epitaxy and characterization of perovskite oxide thin films. J Cryst Growth 227:929-935

[41] Zhang Z, Fang WH, Long R, Prezhdo OV (2019) Exciton dissociation and suppressed charge recombination at $2 \mathrm{~d}$ perovskite edges: key roles of unsaturated halide bonds and thermal disorder. J Am Chem Soc 141(39):15557-15566

Publisher's Note Springer Nature remains neutral with regard to jurisdictional claims in published maps and institutional affiliations. 